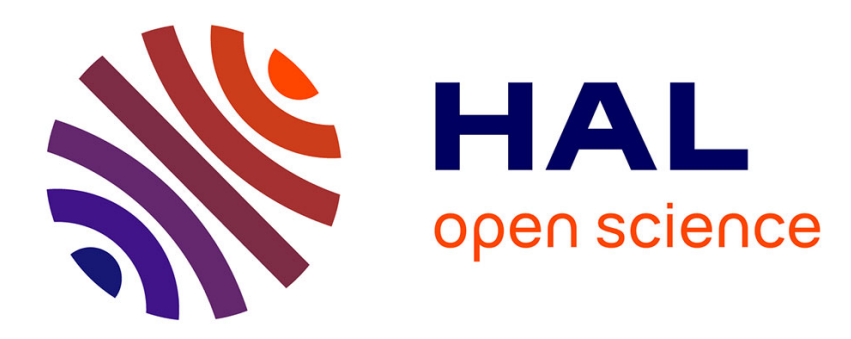

\title{
Etude théorique et expérimentale de l'onde de Rayleigh hélicoïdale
}

\author{
J.-M. Conoir, P. Rembert
}

\section{To cite this version:}

J.-M. Conoir, P. Rembert. Etude théorique et expérimentale de l'onde de Rayleigh hélicoïdale. Journal de Physique IV Proceedings, 1994, 04 (C5), pp.C5-837-C5-840. 10.1051/jp4:19945181 . jpa-00252863

\section{HAL Id: jpa-00252863 https://hal.science/jpa-00252863}

Submitted on 1 Jan 1994

HAL is a multi-disciplinary open access archive for the deposit and dissemination of scientific research documents, whether they are published or not. The documents may come from teaching and research institutions in France or abroad, or from public or private research centers.
L'archive ouverte pluridisciplinaire HAL, est destinée au dépôt et à la diffusion de documents scientifiques de niveau recherche, publiés ou non, émanant des établissements d'enseignement et de recherche français ou étrangers, des laboratoires publics ou privés. 


\title{
Etude théorique et expérimentale de l'onde de Rayleigh hélicoïdale
}

\author{
J.-M. CONOIR et P. REMBERT
}

L.A.U.E., URA 1373 du CNRS, Université du Havre, place R. Schuman, 76610 Le Havre, France

\begin{abstract}
The helical Rayleigh wave propagating on the surface of an elastic cylinder immersed in water is theoretically and experimentaly studied. The experimental detection of this wave is carried out following two different ways. In the first, the position of transducers is defined by the Snell-Descartes laws, and the wave is detected thanks to destructive interferences with the specularly reflected wave. In the second, transducers are separated with regard to the previous position, and the propagation of the wave is detected from maxima of pressure measurement at resonance frequencies.
\end{abstract}

\section{INTRODUCTION}

Lorsqu' on étudie la diffusion en incidence oblique par un cylindre élastique, on est amené à examiner le comportement des ondes d'interface hélicoïdales [1]. Parmi ces ondes, l'onde de Rayleigh tient une place particulière car elle a un comportement différent des autres ondes, dites de Galerie-à-échos, et elle tend en hautes fréquences vers l'onde de Rayleigh de l'interface plane fluide/solide élastique [2]. Quand l'angle d'incidence $\alpha$ varie de 0 (incidence normale) à l'angle de Rayleigh $\underline{\alpha}_{R}$ de l' interface plane, l'onde de Rayleigh passe d'une propagation circonférentielle autour du cylindre à un comportement propagatif suivant son axe, avec une phase intermédiaire où la propagation est hélicoïdale. Dans cette communication, on effectue l'étude théorique des caractéristiques de I' onde de Rayleigh, et des résonances qu'elle crée, quand I' angle d'incidence varie de 0 à $\underline{\alpha}_{R}$. A partir de l'angle critique $\alpha_{T}=\sin ^{-1}\left(C_{1} / C_{T}\right)<\underline{\alpha}_{R}$ où $C_{1}$ est la célérité des ondes dans l'eau et $C_{T}$ celle des ondes transversales dans le cylindre, on montre que I' onde de Rayleigh reste la seule onde d'interface à créer des résonances, ce qui permet son étude expérimentale dans des conditions favorables.

\section{ETUDE THEORIQUE DE L' ONDE DE RAYLEIGH HELICOIDALE}

Lorsque l' angle d'incidence oblique $\alpha$ varie de 0 à $\underline{\alpha}_{R}$, on peut suivre l' évolution des fréquences de résonance attachées à l' onde de Rayleigh ( $c$ : courbe 1 ), de même que la largeur de ces résonances (cf courbe 2). D'une façon générale, on observe que les résonances, créées par un bouclage en phase avec elle même de I' onde de Rayleigh, 
sont larges et donc difficiles à détecter expérimentalement, quel que soit l' angle d'incidence. L'onde de Rayleygh réémet beaucoup d' énergie, qu' elle soit de type circonférentielle ( $\alpha=0$ ), ou hélicoïdale. Par ailleurs, alors que toutes les autres ondes d' interface disparaissent au delà de l' angle critique $\alpha_{T}$ (cf courbe 1 ), l' onde de Rayleigh continue à exister et tend à devenir propagative dans I' axe du cylindre lorsque $\alpha$ tend vers $\underline{\alpha}_{R}$. En effet, La célérité $C_{R}$ de l'onde de Rayleigh est donnée par la relation [1]

$$
C_{1} / C_{R}=\left[\left(n / x_{n}\right)^{2}+\sin ^{2} \alpha\right]^{1 / 2}
$$

où $x_{n}$ est la fréquence de résonance du mode $n$ à l'angle $\alpha$, et l'étude numérique de (1) montre que $\boldsymbol{\alpha}$ et $\mathbf{C}_{R}$ tendent asymptotiquement vers $\underline{\alpha}_{R}$ et la célérité de l' onde de Rayleigh de $l^{\prime}$ interface plane fluide/solide élastique. Par suite, $I$ ' angle de réfraction $\gamma \mathrm{R}$, donné par la relation [1]

$$
\sin \alpha / C_{1}=\sin \gamma_{R} / C_{R}
$$

tend asymptotiquement vers $\pi / 2$ (propagation axiale )quand $\alpha$ tend vers $\underline{\alpha}_{R}$. A titre d'illustration, on a représenté sur la courbe 1 les trajectoires du mode $n=1$ attachées aux ondes d'interface $I=2$ et $p=1$, pour montrer qu'elles disparaissent quand on franchit l'angle $\alpha_{\top}$. Parmi les ondes $d^{\prime}$ 'interface hélicö̈dales, $l=2$ est la première des ondes dites circonférentielles et $p=1$ la première de celles dites guidées [3]. Au delà de l' angle $\alpha_{T}$, la présence de l' onde de Rayleigh hélicoïdale se manifeste par de larges modulations dans la pression diffusée (cf courbe 3 ). Une question reste posée, que devient physiquement l' onde de Rayleigh lorsque $\alpha$ tend vers $\underline{\alpha}_{R}$, et est-ce qu'elle se rattache à un mode de vibration axial connu?

\section{ETUDE EXPERIMENTALE DE L' ONDE DE RAYLEIGH HELICOIDALE}

La mise en évidence expérimentale de l'onde de Rayleigh hélicoïdale est effectuée de deux façons différentes. Soit l'émetteur et le récepteur sont placés suivant les lois de Snell-Descartes (fig 1), et l'onde se manifeste par des interférences destructives avec la réflexion spéculaire (cf courbe 4), soit le récepteur est écarté de la position de SnellDescartes (fig 2), et il reçoit uniquement le rayonnement de l' onde d'interface, rayonnement qu'il détecte par des maximums de pression aux fréquences de résonance (cf courbe 5). Dans la première situation, on compare les résultats expérimentaux avec la pression diffusée (courbes 3 et 4 ), dans la seconde, on compare les fréquences de résonance obtenues expérimentalement avec celles calculées théoriquement.

\section{RESULTATS THEORIQUES ET EXPERIMENTAUX}

Les paramètres élastiques qui caractérisent le diffuseur en acier sont $C_{L}=5790 \mathrm{~m} / \mathrm{s}$ et $C_{T}=3100 \mathrm{~m} / \mathrm{s}$ pour les célérités longitudinale et transversale, $\rho_{S}=7900 \mathrm{~kg} / \mathrm{m} 3$ pour la masse volumique. Avec ces valeurs, l'angle $\alpha_{T}$ vaut $28.3^{\circ}\left(C_{1}=1470 \mathrm{~m} / \mathrm{s}\right)$. Les résultats théoriques et expérimentaux sont présentés pour $\alpha=29^{\circ}>\alpha_{T}$, et sont exprimés en fonction de $k_{1} a$, variable sans dimension souvent appelée fréquence par abus de langage $\left\langle k_{1}=\omega / C_{1}\right.$ où $\omega$ est la pulsation angulaire et a est le rayon du cylindre $)$..

\section{COMPARAISON ENTRE LA THEORIE ET L' EXPERIENCE}

La comparaison entre les calculs théoriques et les expériences pose une difficulté liée au fait que les premiers correspondent à la diffusion par une onde plane d'extension infinie, alors que les secondes sont effectuées avec un émetteur spatialement borné et possédant un spectre angulaire (l'émetteur a une ouverture d' environ $0.5^{\circ}$ autour de son axe d'émission).Comme les résonances attachées à l' onde de Rayleigh varient rapidement avec $\alpha$, une faible erreur dans le positionnement angulaire des transducteurs (effectué à 
partir du temps de vol de la réflexion spéculaire) induit de fortes variations dans les mesures. Par ailleurs, un phénomène de moyennage lié au spectre angulaire est observé sur le positionnement des minimums de pression diffusée.Pour mettre en évidence cet effet, on a moyenné la pression diffusée entre $28.4^{\circ}$ et $28.8^{\circ}$, ce qui déplace très sensiblement la position des minimums de pression (cf tableau). Le choix de la plage angulaire repose sur un double argument : 1 / Compte tenu de la taille des transducteurs et de leur position géométrique, l' influence des ondes émises sous des angles inférieurs à $29^{\circ}$ est prépondérante dans le spectre angulaire ; 2 I on a choisi le meilleur compromis possible entre la théorie et l'expérience.

Pour la comparaison effectuée sur les spectres de diffusion, il ressort que le spectre expérimental est très difficile à comparer directement au spectre théorique, et qu'il est nécessaire de prendre en compte les effets liés à l' ouverture angulaire des transducteurs (cf tableau). En fait, les fréquences de résonance ne coincident pas exactement avec les minimums de la pression diffusée. La comparaison entre les fréquences de résonance semble plus probante, ce qui montre l' intéret des mesures expérimentales effectuées en propagation (cf figure 2). Pour obtenir les mesures en propagation, il est nécessaire d'évaluer la longueur $L_{R}$ qui sépare les "points $d$ ' impact " des transducteurs sur la génératrice du cylindre. Pour cela, on a calculé la distance $d_{R}$ qui sépare les points d'excitation et de réémission de l'onde d'interface hélicoïdale dans le plan contenant les transducteurs et le cylindre (cf figure 3 ), et adapté $L_{R}$ a partir de $d_{R}$. Cette distance $d_{R}$ est donnée par la formule

$$
d_{R}=2 a\left(\pi-\theta_{R}\right) / \cos \gamma_{R}
$$

où

$$
\sin \theta_{R} \cos \gamma_{R}=\left[\left(C_{1} / C_{R}\right)^{2}-\sin ^{2} \alpha\right] 1 / 2
$$

(4)

Malgré les difficultés liées à la sensibilité des mesures expérimentales, et au fait que le modèle théorique ne représente pas exactement les conditions expérimentales, l'accord obtenu entre la théorie et les expériences reste bon, ce qui établit sans ambiguîté le caractère hélicoïdal de la propagation de I' onde de Rayleigh en incidence oblique.

[1] J.-M.CONOIR,P.REMBERT,O.LENOIR,J.-L.IZBICKI, J.Acoust.Soc.Am., 93,3 (1993) 1300-1307.

[2] J.-M.CONOIR,M.TALMANT,J.-L.ROUSSELOT,G.QUENTIN, Actes du 10 ème Congrès Français de Mécanique,Paris, Tome 4(1991)149-152.

[3] J.-L.IZBICKI,G.MAZE, J.RIPOCHE, Traitement du signal,Vol 2 (5), (1985)387-392.

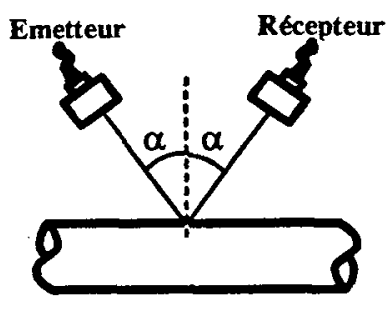

Figure 1 : configuration expérimentale ; transducteurs placés selon les loi de Snell Descartes.

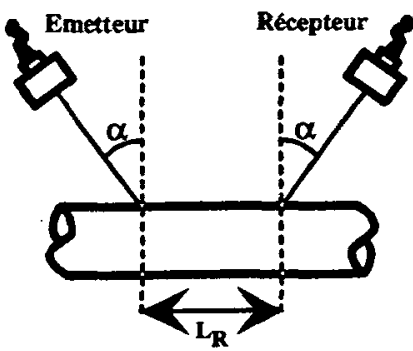

Figure 2 : configuration expérimentale ; transducteurs translatés de $\mathrm{L}_{\mathrm{R}}$ selon l'axe du cylindre par rapport à la configuration de la figure 1.

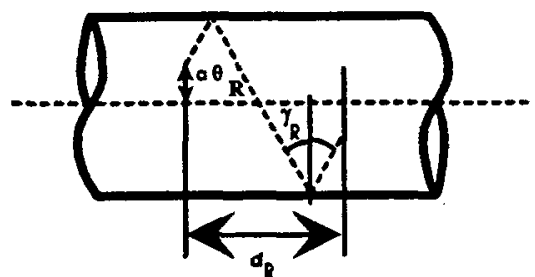

Figure 3 : parcours de l'onde helicoïdale 


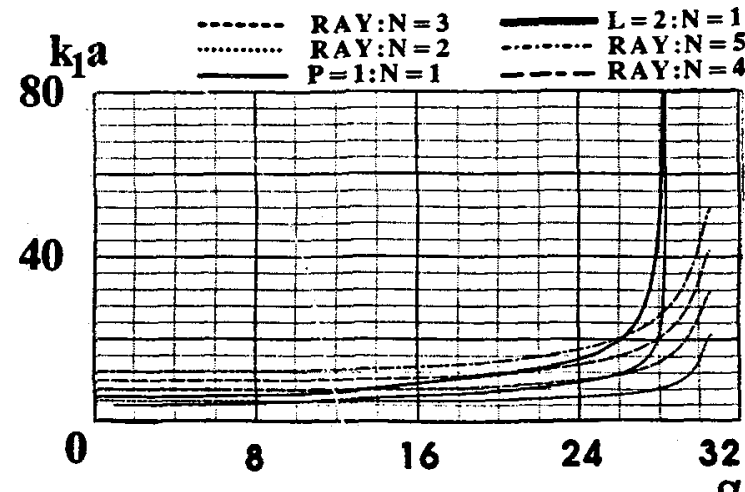

Courbe 1 : evolution des frequences de rtsonance associes $\left.\alpha()^{\circ}\right)$ a l'onde de Rayleigh en fonction ce l'angle d'incidence a
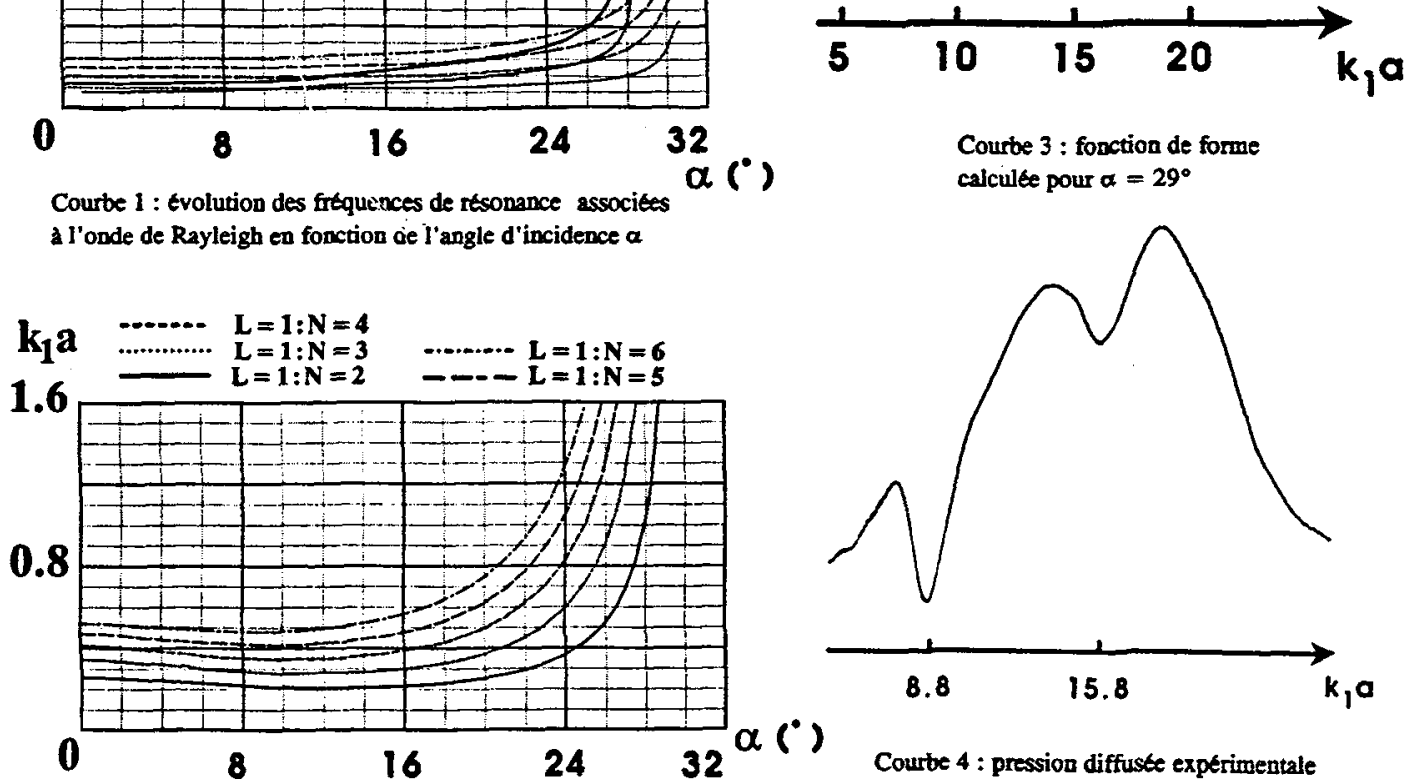

Courbe 3 ; fonction de forme calcule pour $\alpha=29^{\circ}$

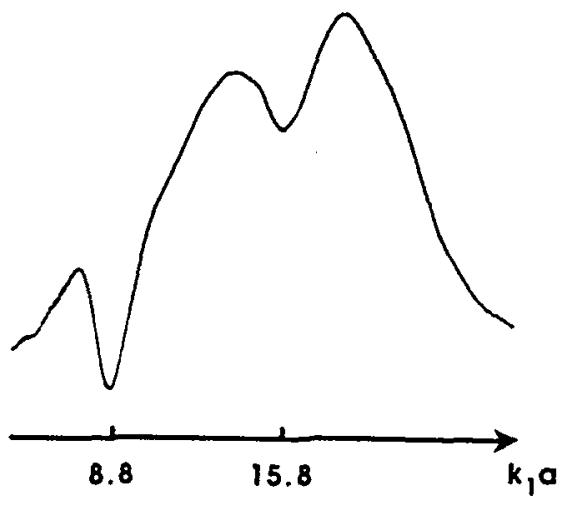

Courbe 4 : pression diffuste experimentale mesurte $\alpha \alpha=29^{\circ}$, transducteurs placés conformement a la figure 1

Courbe 2 : Evolution des largeurs a mi-hauteur des resonances associees a l'onde de Rayleigh en fonction de $a$

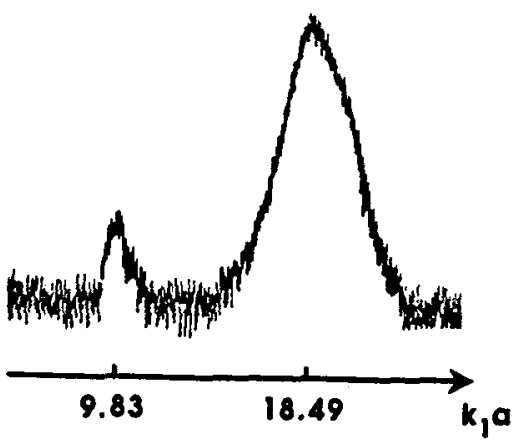

Courbe 5 : pression diffuse experimentale mesurte a $\alpha=29^{\circ}$, transducteurs placts conformement a la figure 2
Tableau : comparaison theorie-experience

\begin{tabular}{|l|c|c|}
\hline Modes & $\mathrm{n}=2$ & $\mathrm{n}=3$ \\
\hline $\begin{array}{l}\text { fréquences de résonance } \\
\text { thériques }\end{array}$ & 9.95 & 18 \\
\hline $\begin{array}{l}\text { fréquences de résonance } \\
\text { expérimentales (courbe 5) }\end{array}$ & 9.83 & 18.49 \\
\hline $\begin{array}{l}\text { minimums de in pression diffusée } \\
\text { thérique }\end{array}$ & 9.6 & 17.9 \\
\hline $\begin{array}{l}\text { minimums de b pression diffusé } \\
\text { moyenné }\end{array}$ & 8.8 & 16.2 \\
\hline $\begin{array}{l}\text { minimums de la pression diffusée } \\
\text { expérimentale (courbe 4) }\end{array}$ & 8.8 & 15.8 \\
\hline
\end{tabular}

